\title{
Apatinib for the treatment of gastric cancer
}

\author{
Giandomenico Roviello, Andrea Ravelli, Anna Ida Fiaschi, Maria Rosa \\ Cappelletti, Angela Gobbi, Chiara Senti, Laura Zanotti, Karol Polom, Andrew \\ R Reynolds, Stephen B. Fox \& Daniele Generali
}

To cite this article: Giandomenico Roviello, Andrea Ravelli, Anna Ida Fiaschi, Maria Rosa Cappelletti, Angela Gobbi, Chiara Senti, Laura Zanotti, Karol Polom, Andrew R Reynolds, Stephen B. Fox \& Daniele Generali (2016): Apatinib for the treatment of gastric cancer, Expert Review of Gastroenterology \& Hepatology, DOI: 10.1080/17474124.2016.1209407

To link to this article: http://dx.doi.org/10.1080/17474124.2016.1209407

Accepted author version posted online: 04 Jul 2016. Published online: 04 Jul 2016.

Submit your article to this journal $₫$

Џll Article views: 2

Q View related articles $₫$

View Crossmark data $₫$ 
Publisher: Taylor \& Francis

Journal: Expert Review of Gastroenterology \& Hepatology

DOI: $10.1080 / 17474124.2016 .1209407$

\section{Apatinib for the treatment of gastric cancer}

Giandomenico Roviello ${ }^{1,2}$, Andrea Ravelli ${ }^{3}$, Anna Ida Fiaschi ${ }^{4}$, Maria Rosa Cappelletti ${ }^{2}$, Angela Gobbi ${ }^{2}$, Chiara Senti ${ }^{2}$, Laura Zanotti ${ }^{2}$, Karol Polom ${ }^{5}$, Andrew R Reynolds ${ }^{6}$, Stephen B. Fox ${ }^{7}$, Daniele Generali ${ }^{2,8}$

1) Department of Molecular and Translational Medicine, University of Brescia, 25123 Brescia, Italy

2) Unit of Molecular therapy and Pharmacogenomic, ASST Cremona, Viale Concordia 1, 26100 Cremona, Italy

3) Section of Experimental Oncology, Department of Clinical and Experimental Medicine, University of Parma, Via Università, 12, 43121 Parma, Italy

4) Pharmacology Unit, University of Siena, Siena, Italy

5) Department of Medical, Surgical and Neuroscience; Unit of General and Minimally Invasive Surgery, University of Siena, Viale Bracci 11, 53100 Siena, Jtaly

6) Tumour Biology Team, Breast Cancer Now Research Centre, The Institute of Cancer Research, London SW3 6JB, England

7) Department of Pathology, Peter Mac Callum Cancer Centre, and Department of Pathology, University of Melbourne, Australia

8) Department of Medical, Surgery and Health Sciences, University of Trieste, Piazza Ospitale 1, 34129 Trieste, Italy

Address for correspondence: Giandomenico Roviello MD

Department of Molecular and Translational Medicine, University of Brescia, Viale Europa 11, 25124 Brescia, Italy. E-mail: giandomenicoroviello@gmail.com

\section{Summary}

Apatinib, a small-molecule inhibitor of vascular endothelial growth factor receptor 2, has demonstrated encouraging anti-cancer activity in gastric cancer within both in vitro and in vivo models. Its therapeutic efficacy, tolerability and safety have been evaluated in one Phase II and one 
Phase III study in metastatic/advanced gastric cancer. In this review, we focus on the mechanism of action of apatinib, its pharmacokinetic profile and its clinical activity in the treatment of advanced/metastatic gastric cancer.

Keywords: Gastric cancer, Apatinib, VEGFR2, angiogenesis, chemotherapy

\section{Introduction}

Gastric cancer (GC) is the fifth most common cancer worldwide and, despite improvements in the treatment of this disease, GC is the third most common cancer-related cause of death in the world [1]. It is estimated that in the entire world population, about 1 million people are diagnosed with GC annually, and approximately half of these cases are in China [1]. In the metastatic setting, chemotherapy provides only palliation of symptoms and improvement in the quality of life compared to best supportive care, and has only a modest impact on survival [2,3]. In general, regimens which combine a fluoropyrimidine with a platinum-based agent are the most widely accepted as a standard first-line therapy [4,5]. A three-drug chemotherapy regimen, with the addition of an anthracycline or docetaxel, may represent a possible option for patients who can tolerate more aggressive chemotherapy. Second lines of therapy may include irinotecan and taxanes, however their use is limited only to patients with an adequate performance status. Unfortunately, after failure of second-line treatment, the results of further lines of chemotherapy are disappointing (based on results in human epidermal receptor 2 (HER-2) positive GC), and no chemotherapeutic agents showed a clear benefit in prolonging survival [6]. Therefore, the discovery of novel, effective and safe treatment options is an unmet clinical need for metastatic GC.

Apatinib, also known as YN968D1, is a novel receptor tyrosine kinase inhibitor selectively targeting the intracellular ATP-binding site of vascular endothelial growth factor receptor 2 (VEGFR2) [7,8]. Evidence that apatinib treatment provides a clinical benefit in patients with metastatic GC is now available. This review will examine the role of apatinib in GC, with 
references to its proposed mechanism of action, as well as its efficacy, safety, and tolerability in clinical trials.

\section{Body of review}

\subsection{Overview of the market}

Treatment of metastatic GC is still a real clinical challenge. Although several chemotherapeutic agents, including platinum-based compounds (cisplatin and oxaliplatin), fluoropyrimidines (5fluorouracil; capecitabine and S-1 in Asiatic countries), taxanes (docetaxel and paclitaxel), and the anthracyclines, such as epirubicin, are currently available for first- and second-line therapy in metastatic disease, the prognosis still remains poor with a low percentage of long term survivors and an overall survival that does not exceed 12 months $[4,9,10]$. More recently, biological therapies have become available for the treatment of metastatic GC [11]. Trastuzumab, a monoclonal antibody against HER-2, is approved for the $20 \%$ of patients with HER-2 positive GC tumours [12]. Moreover, ramucirumab, a monoclonal antibody targeted against VEGFR2, has shown a survival benefit as a second line treatment option in those metastatic GC patients who progressed on fluoropyrimidine- or platinum-based first-line chemotherapy [12-14].

Unfortunately, very few data are available on the use of chemotherapy in the third-line setting: only taxane or irinotecan-based regimens have shown modest effects on survival with a very low rate of response [15-16]. Recent reports suggest that regorafenib, a pan-receptor tyrosine kinase inhibitor, may have some activity in GC [17]. The INTEGRATE study showed a progression free survival advantage for regorafenib compared to placebo in refractory advanced GC patients who had already progressed after first and second line therapy [17]. Due to these newly available treatment options, the number of patients with metastatic GC who have been treated with several lines of therapy will increase. Amongst the newly available biological therapies available for the treatment of GC, apatinib has shown promising activity [18] and will be the subject of this review. 


\subsection{Introduction to the drug}

\subsubsection{Chemistry}

Apatinib is an orally bioavailable small-molecule anti-angiogenic agent, with a molecular weight of 493.58 Da, that potently inhibits VEGFR2, whilst also have some activity for inhibition of the platelet-derived growth factor receptor- $\beta$ (PDGFR- $\beta$ ), c-kit, and c-src [18]. The molecular formula of apatinib is $\mathrm{C} 25 \mathrm{H} 27 \mathrm{~N} 5 \mathrm{O} 4 \mathrm{~S}$, and chemical formula is N-[4-(1-cyano-cyclopentyl)phenyl]-2-(4pyridylmethyl) amino-3-pyridine carboxamide, which is shown in [19] Figure 1.

\subsubsection{Mechanism of action}

VEGFR2 is stimulated by VEGF molecules that induce dimerization of the receptor and a subsequent auto-phosphorylation. VEGFR2 activation stimulates several downstream signalling pathways, including the Raf/MEK/ERK1/2, the p38-MAPK and the PI3K/AKT/mTOR pathway $[20,21]$. VEGF stimulation of the receptor induces the sprouting of new blood vessels from preexisting vessels [22]. The mechanism of action of apatinib is mediated by its binding to the intracellular ATP-binding site of VEGFR2 receptor (Figure 2) which blocks its phosphorylation and subsequently the downstream pro-angiogenic signalling. Apatinib displays both anti-angiogenic and anti-tumour activity in preclinical models, including a stomach cancer xenograft model [18].

\subsubsection{Pharmacokinetics and metabolism}

The pharmacokinetics and metabolism of apatinib have been studied. After administration of apatinib to human subjects, the time to maximum plasma concentration level was $\sim 4$ hours after dosing and the mean half-life was 9 hours [23]. In three different dosing regimens $(500,750$ and 800), a dose-dependent $\mathrm{C}_{\max }$ and $\mathrm{AUC}_{24}$ were observed with $\mathrm{C}_{\max }$ of 1,521,2,379, and 2,833 $\mathrm{ng} / \mathrm{mL}$ and $\mathrm{AUC}_{24}$ of $11,295,18,172$, and 21,975 $\mathrm{ng} \mathrm{h} / \mathrm{mL}$, respectively [24]. Further analysis suggested no accumulation of drug following 56 days of once-a-day administration of $750 \mathrm{mg}$ apatinib [23]. The primary routes of apatinib biotransformation include E- and Z-cyclopentyl-3- 
hydroxylation, N-dealkylation, pyridyl-25-N-oxidation, 16-hydroxylation, dioxygenation, and Oglucuronidation after 3-hydroxylation 15DDT. The major circulating metabolite was the pharmacologically inactive E-3-hydroxy-apatinib-O-glucuronide (M9-2) [24]. The primary metabolisers of apatinib are CYP3A4/5 and UGT2B7 is the main enzyme responsible for M9-2 formation [25]. The total recovery of the administered dose was $76.8 \%$ within 96 hours post-dose, with $69.8 \%$ and $7.02 \%$ of the administered dose excreted in feces and urine, respectively [24,25].

\subsection{Clinical efficacy}

\subsubsection{Phase I study}

The clinical development of apatinib has been carried out mostly in China. In 2010, a phase I study was performed to determine the pharmacological activity and the maximum tolerated dose (MTD) of apatinib for patients with advanced solid tumors [23]. Thirty-four patients enrolled in the study had gastrointestinal tract-related tumors. The study established that the MTD for apatinib was 850 mg once daily. Moreover, a significant antitumor activity was also observed in patients with measurable lesions; the 7 patients achieving partial response were mainly those with GC tumors. The most frequently observed drug-related adverse events were hypertension, proteinuria, and hand-foot syndrome (which are all common drug class effects of oral VEGFR2 tyrosine kinase inhibitors) and they were clinically manageable.

\subsubsection{Phase II study}

A single phase II study investigated apatinib versus placebo in heavily pre-treated metastatic GC patients [26]. All the enrolled patients had progressed after two lines of therapy and about $30 \%$ were treated with $\geq 3$ lines of prior therapy. Two schedules of apatinib were evaluated: the $850 \mathrm{mg}$ once daily regimen and the $425 \mathrm{mg}$ twice daily regimen. The primary end-point of this study was progression free survival (PFS). One hundred and forty one patients were divided as follow: 48 in 
the placebo group, 47 in the arm with $850 \mathrm{mg}$ apatinib once daily and 46 in the arm with $425 \mathrm{mg}$ apatinib twice daily. The percentage of patients receiving at least two cycles of treatment was $50 \%$ in the placebo group, $74.5 \%$ in the $850 \mathrm{mg}$ group and $69.6 \%$ in the $425 \mathrm{mg}$ group [26]. Apatinib treatment significantly improved the PFS when compared with placebo. PFS was 3.67 months and 3.20 months for patients who received apatinib $850 \mathrm{mg}$ once daily and $425 \mathrm{mg}$ twice daily, respectively, and 1.40 months for patients who received placebo. Multivariate Cox regression analysis showed that PFS was significantly different between apatinib versus placebo without any significant difference between the two schedules of apatinib. The median OS values were 4.83 months and 4.27 months, for patients who received $850 \mathrm{mg}$ once daily apatinib and $425 \mathrm{mg}$ twice daily apatinib, respectively, and 2.50 months in the placebo group. The overall response rate and disease control rate favoured the arms containing apatinib versus placebo.

\subsubsection{Phase III studies}

Based on the results of the phase II trial [26], a randomized, double-blind, placebo-controlled phase III trial of apatinib in heavily pre-treated advanced or metastatic gastric or gastroesophageal junction cancer was conducted [27]. Two hundred and sixty seven patients were enrolled; 176 were assigned to the apatinib group and 91 were assigned to the placebo group. The primary end-points were OS and PFS. Patients in the apatinib group received a median of 2.9 cycles of therapy, and $72 \%$ of patients were treated with two or more cycles of therapy. Patients in the placebo group received a median of 1.89 cycles of placebo, and $58 \%$ of patients were treated with two or more cycles of placebo $(\mathrm{P}=0.0028)$ [27]. The disease control rate was significantly improved with apatinib compared to placebo $(31.82 \%$ versus $10.99 \% ; \mathrm{P}<0.001)$. The overall response rate was $1.7 \%$ with apatinib versus $0 \%$ with placebo. The median PFS was also significantly longer (2.6 vs 1.8 months; $\mathrm{HR}=0.444 ; \mathrm{P}<0.001)$. The median OS was 6.5 months with apatinib versus only 4.7 months with placebo $(\mathrm{HR}=0.709,95 \% \mathrm{CI}, 0.537-0.595, \mathrm{P}=0.149)$. The quality of life was evaluated using the European Organization for Research and Treatment of Cancer Quality of Life Questionnaire Core 30 (EORTCQLQ-C30) at three different time points (baseline and end of cycles 
two and three). The analysis showed no significant differences between the two groups at any time point $(\mathrm{P}>0.05)$.

\section{Safety and tolerability}

In the phase I trial [23], the most frequently observed drug-related adverse events with apatinib were hypertension $(69.5 \%, 29$ grade $1-2$ and three grade $3-4)$, proteinuria $(47.8 \%, 16$ grade $1-2$ and six grade 3-4), and hand-foot syndrome (45.6\%, 15 grade 1-2 and six grade 3-4). Eighteen patients had a dose reduction due to different toxicities. In the phase II trial, grade 3-4 adverse events occurred in more than 5\% of patients [26] and the most frequent were: hand-foot syndrome, hypertension, thrombocytopenia, anemia, diarrhea, and liver toxicity (mainly, elevation of aminotransferase and bilirubin levels). The most frequently observed grade 3-4 adverse events were hand-foot syndrome and hypertension detected in the $10 \%$ of the patients. Fatigue was a common adverse event, affecting a total of $10.4 \%, 17.0 \%$, and $15.2 \%$ of patients receiving placebo, apatinib $850 \mathrm{mg}$ once daily, or apatinib $425 \mathrm{mg}$ twice daily, respectively.

In the phase III trial [27], dose modifications resulting from toxicity were more common in the apatinib arm compared to the placebo arm $(21.0 \%$ versus $3.3 \%$, respectively). The main reasons for dose reduction were hand-foot syndrome, proteinuria, and hypertension. A grade 3-4 hand-foot syndrome occurred in $8.5 \%$ and $0 \%$ of patients in the apatinib and placebo group, respectively $(\mathrm{P}=0.0032)$. Grade $3-4$ hypertension and proteinuria occurred in $4.5 \%$ and $2.3 \%$ of patients in the apatinib group, but was $0 \%$ in the placebo group. Grade 3-4 neutropenia, transaminase elevation and hypophosphatemia were also increased in the apatinib arm. Details of adverse events from these studies are provided in Table 1. 


\section{Regulatory affairs}

Based on the data from the phase III study, apatinib was approved in October 2014 by the China Food and Drug Administration (CFDA) for patients with metastatic gastric or gastroesophageal junction adenocarcinoma after the second-line of chemotherapy [28].

\section{Conclusion}

Apatinib is a novel, orally bioavailable small-molecule tyrosine kinase inhibitor of VEGFR2 that appears to be a promising agent in the treatment of a variety of tumor types including metastatic GC. However, although the initial clinical results are promising, it should be stated that the small number of patients recruited in these clinical trials does preclude definitive conclusions at the current time. Nonetheless, apatinib compared to placebo has been shown to improve overall survival, progression free survival and response rate in heavily pre-treated patients (i.e. those receiving more than 2 lines of prior therapy). Tumor angiogenesis is a crucial process in cancer development and progression [29], especially in gastro-intestinal tumors [30], thus targeting it could be an interesting and beneficial therapeutic option. At this point, it is worth recapping what is known regarding other anti-angiogenic agents in GC. The monoclonal anti-VEGF antibody bevacizumab, in combination with chemotherapy, did not met its primary endpoint to improve survival in metastatic GC [31]. Ramucirumab is a humanized monoclonal antibody against VEGFR2 which prevents VEGF ligand binding and receptor-mediated pathway activation in endothelial cells [11]. Ramucirumab is approved for patients with advanced gastric or gastroesophageal junction cancer that experienced progression after first-line chemotherapy [11]. Approval was based on gains in overall survival when the drug was administered either alone or in combination with paclitaxel in phase III studies [11]. Of note, although ramucirumab and apatinib target the same signalling pathway, they have different mechanisms of action. However, their efficacy in GC suggests an important role for VEGF receptor signalling in GC. Nonetheless the clinical advantage seems modest (around two months gain in both progression free survival and 
overall survival). Since apatinib is the first tyrosine-kinase inhibitor shown to be effective in metastatic GC, the continued clinical exploitation of this drug for metastatic GC is warranted.

\section{Expert commentary}

It should be noted that although the clinical studies performed so far support the use of apatinib in GC, this may be mainly due to the real absence of an efficacious treatment as a comparator. Therefore, the real clinical efficacy of apatinib is still to be weighed in larger trials.

It is also important to consider whether a subset of GC patients can be selected which optimally respond to apatinib. The Cancer Genome Atlas Research Network (TCGA) has demonstrated a subset of GC with a recurrent amplification of the VEGF-A gene and with an elevated expression of angiogenesis-related pathways [31-34]. In addition, several trials showed that the expression of VEGFR2 is related to a poor prognosis in patients with metastatic GC [35]. Given the molecular evidence that the VEGF pathway is upregulated in only a subset of GC cancers, it may be beneficial to pursue predictive biomarkers for anti-VEGF pathway therapies in this cancer. This would help in selecting the right patients to treat with apatinib. One potential candidate for such a biomarker is the expression level or activation status of VEGFR2 in GC. In this context, a study of apatinib in patients with breast cancer showed that both hypertension and high expression of phosphorylated VEGFR2 could serve as potential biomarkers for treatment efficacy [36]. Unfortunately, as yet, there is no definitive biomarker data for apatinib in GC.

\section{Five-year view}

The efficacy and safety of apatinib in metastatic GC will be evaluated in an on-going phase IV study (NCT02426034) already started in April 2015. A total of 2000 patients are planned to be enrolled. Undoubtedly, this large study will provide important information as to the clinical role of apatinib in the second line treatment of GC. Additional studies will investigate the role of apatinib as a maintenance therapy after the first line of treatment in GC. Apatinib will be also investigated in combination with chemotherapeutic agents such as S-1 in the first-line of treatment or S- 
1/paclitaxel based chemotherapy in the conversion therapy of metastatic GC [37]. Details of these trials are provided in Table 2.

\section{Key issues}

- Apatinib is a novel receptor tyrosine kinase inhibitor selectively targeting the intracellular ATP-binding site of the vascular endothelial growth factor receptor 2 (VEGFR2)

- Apatinib compared to placebo has been shown to improve survival, progression free survival and response rate in heavily pre-treated GC patients.

- The toxicities of apatinib were tolerable or clinically manageable. Hypertension, hand-andfoot syndrome, proteinuria, fatigue, anorexia, and elevated aminotransferase were the most commonly observed adverse events.

\section{Information resources}

Basic information on the chemistry and properties of apatinib can be obtained from the online Salix Pharmaceuticals drug information or from the PubChem website. For an overview of the diagnosis and management of GC, please see the American College of Gastroenterology. For references directly pertaining to the action or use of apatinib please see the recommended references below. 


\section{References}

\section{Reference annotations}

* Of interest

** Of considerable interest

[1] Ferlay J, Soerjomataram I, Dikshit R, et al. Cancer incidence and mortality worldwide: sources, methods and major patterns in GLOBOCAN 2012. Int J Cancer. 2015;136(5):E359-386.

[2] Glimelius B, Hoffman K, Haglund U, et al. Initial or delayed chemotherapy with best supportive care in advanced gastric cancer. Ann Oncol 1994;5:189-190.

[3] Pyrhonen S, Kuitunen T, Nyandoto P, Kouri M. Randomised comparison of fluorouracil, epidoxorubicin and methotrexate (FEMTX) plus supportive care with supportive care alone in patients with non-resectable gastric cancer. Br J Cancer 1995; 71:587-591.

* [4] Wagner AD, Unverzagt S, Grothe W, Kleber G, Grothey A, Haerting J, Fleig WE. Chemotherapy for advanced gastric cancer. Cochrane Database Syst Rev 2010; (3): CD004064.

\section{Chemotherapy significantly improves survival in comparison to best supportive care.}

[5] Petrioli R, Roviello G, Zanotti L, Roviello F, Polom K, Bottini A, Marano L, Francini E, Marrelli D, Generali D. Epirubicin-based compared with docetaxel-based chemotherapy for advanced gastric carcinoma: A systematic review and meta-analysis. Crit Rev Oncol Hematol. 2016 Jun;102:82-8.

[6] National Comprehensive Cancer Network: NCCN Clinical Practice Guidelines in Oncology: Gastric Cancer (version 2.2015). http://www.nccn.org/professionals/physician_gls/pdf/gastric.pdf.

[7] Tian, S., et al., YN968D1 is a novel and selective inhibitor of vascular endothelial growth factor receptor-2 tyrosine kinase with potent activity in vitro and in vivo. Cancer Sci, 2011. 102(7): p. 1374-80.

[8] Wilhelm, S.M., et al., BAY 43-9006 exhibits broad spectrum oral antitumor activity and targets the RAF/MEK/ERK pathway and receptor tyrosine kinases involved in tumor progression and angiogenesis. Cancer Res, 2004. 64(19): p. 7099-109.

[9] Petrioli R. et al. Sequential treatment with epirubicin, oxaliplatin and 5FU (EOF) followed by docetaxel, oxaliplatin and 5FU (DOF) in patients with advanced gastric or gastroesophageal cancer: a single-institution experience. Cancer Chemother Pharmacol, 2015. 75(5): p. 941-7.

[10] Lordick, F. et al, Unmet needs and challenges in gastric cancer: the way forward. Cancer Treat Rev, 2014. 40(6): p. $692-700$.

[11] Roviello G, Polom K, Petrioli R, Marano L, Marrelli D, Paganini G, Savelli V, Generali D, De Franco L, Ravelli A, Roviello F. Monoclonal antibodies-based treatment in gastric cancer: current status and future perspectives. Tumour Biol. 2015 Nov 13.

*[12] Bang Y.J. et al. Trastuzumab in combination with chemotherapy versus chemotherapy alone for treatment of HER2-positive advanced gastric or gastro-oesophageal junction cancer (ToGA): a phase 3, open-label, randomised controlled trial. Lancet, 2010. 376(9742): p. 687-97.

Trastuzumab in combination with chemotherapy is effective for patients with HER2-positive advanced gastric or gastro-oesophageal junction cancer

[13] de Mello R.A. A.M. Marques, and A. Araujo, HER2 therapies and gastric cancer: a step forward. World J Gastroenterol, 2013. 19(37): p. 6165-9. 
[14] Janjigian, Y.Y. et al. Prognosis of metastatic gastric and gastroesophageal junction cancer by HER2 status: a European and USA International collaborative analysis. Ann Oncol, 2012. 23(10): p. 2656-62.

[15] Kang EJ, Im SA, Oh DY, et al: Irinotecan combined with 5-fluorouracil and leucovorin third-line chemotherapy after failure of fluoropyrimidine, platinum, and taxane in gastric cancer: Treatment outcomes and a prognostic model to predict survival. Gastric Cancer 16: 581-589, 2013

[16] Park JS, LimJY, Park SK, et al: Prognostic factors of second and third line chemotherapy using 5-fu with platinum, irinotecan, and taxane for advanced gastric cancer. Cancer Res Treat 43:236-243, 2011.

[17] Pavlakis N, Sjoquist KM, Tsobanis E, Martin AJ et al. INTEGRATE: A randomized, phase II, double-blind, placebo-controlled study of regorafenib in refractory advanced oesophagogastric cancer (AOGC): A study by the Australasian Gastrointestinal Trials Group (AGITG)_Final overall and subgroup results. J Clin Oncol (Meeting Abstracts) May 2015 vol. 33 no. 15_suppl 4003.

[18] Tian S, Quan H, Xie C, et al. YN968D1 is a novel and selective inhibitor of vascular endothelial growth factor receptor-2 tyrosine kinase with potent activity in vitro and in vivo. Cancer Sci. 2011;102(7):1374-1380.

[19] Chen PI.-A.L., Lou L, Sun P, Yuan K:, VEGFr inhibitor YN968D1 xenograft dose response studies against human colon cancer Ls174t and HT29. Proc Amer Assoc Cancer Res 2006, 47, abst 1764.

[20] Hicklin D.J. and L.M. Ellis, Role of the vascular endothelial growth factor pathway in tumor growth and angiogenesis. J Clin Oncol, 2005. 23(5): p. 1011-27.

[21] Holmes K. et al. Vascular endothelial growth factor receptor-2: structure, function, intracellular signalling and therapeutic inhibition. Cell Signal, 2007. 19(10): p. 2003-12.

[22] Longo, R. and G. Gasparini, Challenges for patient selection with VEGF inhibitors. Cancer Chemother Pharmacol, 2007. 60(2): p. 151-70.

**[23] Li J, Zhao X, Chen L, et al. Safety and pharmacokinetics of novel selective vascular endothelial growth factor receptor-2 inhibitor YN968D1 in patients with advanced malignancies. BMC Cancer. 2010;10:529.

YN968D1 (Apatinib) selectively inhibits phosphorylation of VEGFR2 and tumor angiogenesis in preclinical models. In patients, the recommended dose of $750 \mathrm{mg}$ once daily was well tolerated. Encouraging anti-tumor activity across a broad range of malignancies warrants further evaluation in selected populations.

[24] Ding J, Chen X, Gao Z, et al. Metabolism and pharmacokinetics of novel selective vascular endothelial growth factor receptor-2 inhibitor apatinib in humans. Drug Metab Dispos. 2013;41(6):1195-1210.

[25] Zhang H. Apatinib for molecular targeted therapy in tumor. Drug Des Devel Ther. 2015 Nov 13;9:6075-81.

**[26] Li, J et al, Apatinib for chemotherapy-refractory advanced metastatic gastric cancer: results from a randomized, placebo-controlled, parallel-arm, phase II trial. J Clin Oncol, 2013. 31(26): p. 3219-25.

Apatinib showed improved PFS and OS in heavily pretreated patients with metastatic GC who had experienced treatment failure with two or more chemotherapy regimens.

**[27] Li J, Qin S, Xu J, et al; Randomized, Double-Blind, Placebo-Controlled Phase III Trial of Apatinib in Patients With Chemotherapy-Refractory Advanced or Metastatic Adenocarcinoma of the Stomach or Gastroesophageal Junction. J Clin Oncol. 2016 Feb 16.

Apatinib treatment significantly improved OS and PFS with an acceptable safety profile in patients with advanced gastric cancer refractory to two or more lines of prior chemotherapy.

[28] Apatinib got CFDA approval. http://www.xinyaohui.com/news/201502/05/ 5059.html

[29] Ferrara N, Gerber HP, LeCouter J. The biology of VEGF and its receptors. Nat Med 2003; 9: 669-676. 
[30] Ferrara N. Vascular endothelial growth factor as a target for anticancer therapy. Oncologist 2004;9 Suppl 1:2-10.

*[31] Ohtsu A, Shah MA, Van Cutsem E, et al: Bevacizumab in combination with chemotherapy as first-line therapy in advanced gastric cancer: A randomized, double-blind, placebo-controlled phase III study. J Clin Oncol 29:3968-3976, 2011

Bevacizumab added to chemotherapy did not reach its primary objective of improved overall survival. However, adding bevacizumab to chemotherapy was associated with a significant increase in progression-free survival and overall response rate in the first-line treatment of advanced gastric cancer.

[32] Cancer Genome Atlas Research N. Comprehensive molecular characterization of gastric adenocarcinoma. Nature, 2014. 513(7517): p. 202-9.

[33] Aprile G. et al. Angiogenic inhibitors in gastric cancers and gastroesophageal junction carcinomas: A critical insight. Crit Rev Oncol Hematol, 2015. 95(2): p. 165-78.

[34] Fontana EF. Sclafani, and D. Cunningham, Anti-angiogenic therapies for advanced esophago-gastric cancer. Indian J Med Paediatr Oncol, 2014. 35(4): p. 253-62.

[35] Roviello G. et al. Angiogenesis inhibitors in gastric and gastroesophageal junction cancer. Gastric Cancer, 2015.

[36] Fan M, Zhang J, Wang Z, et al: Phosphorylated VEGFR2 and hypertension: Potential biomarkers to indicate VEGF-dependency of advanced breast cancer in anti-angiogenic therapy. Breast Cancer Res Treat 143:141-151, 2014.

[37] Roviello G, Roviello F, Polom K, Generali D. Apatinib in metastatic gastric cancer: can paclitaxel make the difference? Anticancer Drugs. 2016 Jun 15.

Financial and competing interests disclosure

The authors have no relevant affiliations or financial involvement with any organization or entity with a financial interest in or financial conflict with the subject matter or materials discussed in the manuscript. This includes employment, consultancies, honoraria, stock ownership or options, expert testimony, grants or patents received or pending, or royalties. 
Figure 1. Chemical structure of apatinib.

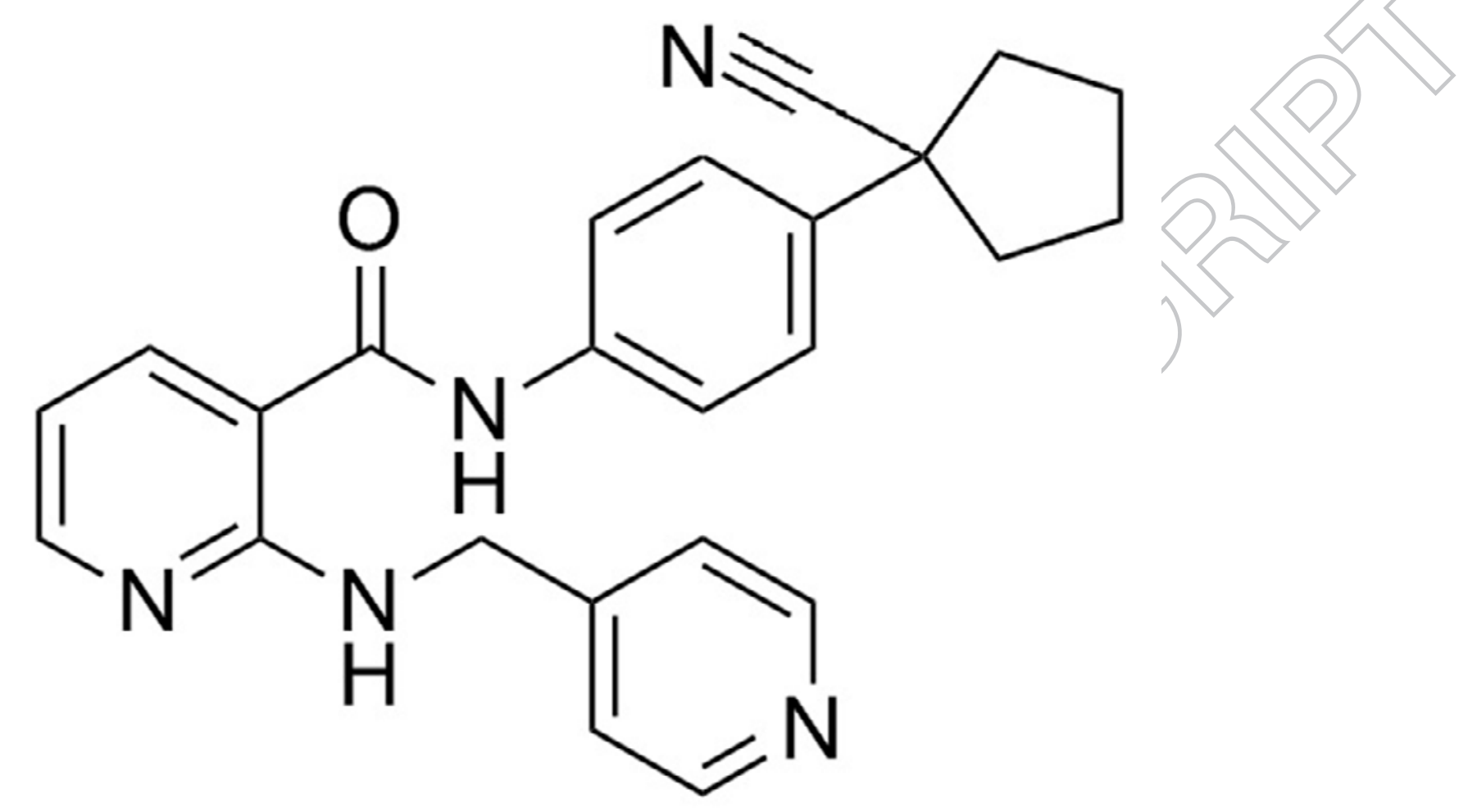


Figure 2. Mechanism of action of apatinib

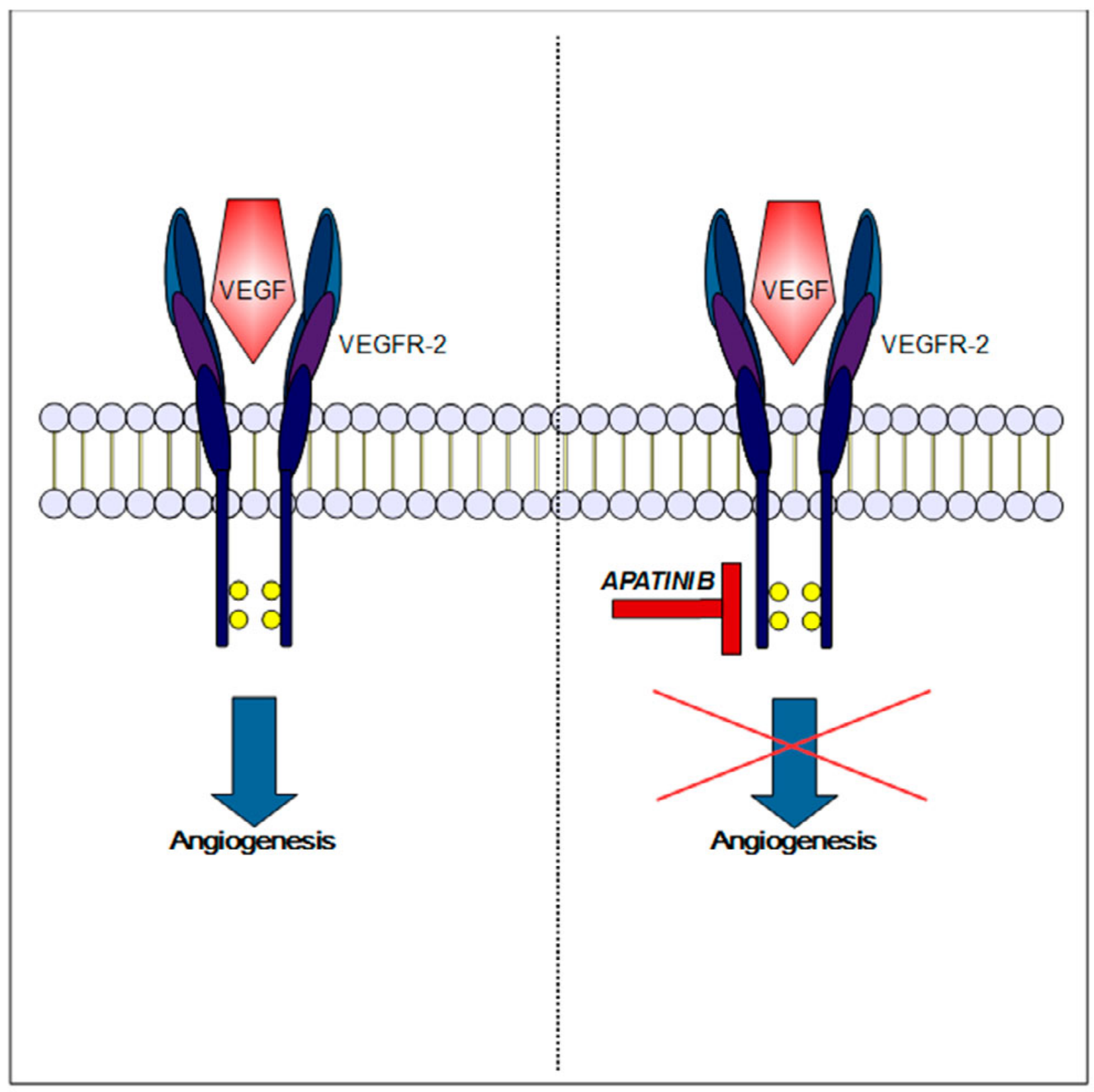


Table 1. Adverse events in clinical trials of apatinib for gastric cancer.

\begin{tabular}{|c|c|c|c|c|c|c|c|c|c|c|}
\hline \multirow{4}{*}{ Adverse event } & \multicolumn{10}{|c|}{$\begin{array}{c}\text { Numbers } \\
(\%)\end{array}$} \\
\hline & \multicolumn{6}{|c|}{ Li et al. 2013} & \multicolumn{4}{|c|}{ Li et al. 2016} \\
\hline & \multicolumn{2}{|c|}{$\begin{array}{l}\text { Apatinib } 425 \mathrm{mg} \\
\quad(\mathrm{n}=46)\end{array}$} & \multicolumn{2}{|c|}{$\begin{array}{c}\text { Apatinib } 850 \mathrm{mg} \\
(\mathrm{n}=47)\end{array}$} & \multicolumn{2}{|c|}{$\begin{array}{c}\text { Placebo } \\
(\mathrm{n}=48)\end{array}$} & \multicolumn{2}{|c|}{$\begin{array}{l}\text { Apatinib } \\
(\mathrm{n}=176)\end{array}$} & \multicolumn{2}{|c|}{$\begin{array}{c}\text { Placebo } \\
(\mathrm{n}=91)\end{array}$} \\
\hline & Any grade & Grade 3-4 & Any grade & Grade 3-4 & Any grade & Grade 3-4 & Any grade & Grade 3-4 & Any grade & Grade 3-4 \\
\hline Leukopenia & $18(39)$ & $2(4.3)$ & $23(49)$ & $0(0)$ & $4(8.3)$ & $2(4,2)$ & $71(40.3)$ & $3(1.7)$ & $8(8.8)$ & $0(0)$ \\
\hline Neutropenia & $15(32.6)$ & $2(4.3)$ & $18(38.3)$ & $1(2.1)$ & $3(6.3)$ & $2(4.2)$ & $66(37.5)$ & $10(5.7)$ & $9(9.9)$ & $1(1.1)$ \\
\hline Anemia & $8(17.4)$ & $3(6.5)$ & $9(19.1)$ & $1(2.1)$ & $9(18.8)$ & $3(6.3)$ & $44(25)$ & $11(6.3)$ & $22(24.2)$ & $4(4.4)$ \\
\hline Thrombocytopenia & $18(39.1)$ & $2(4.3)$ & $14(29.8)$ & $2(4.3)$ & $6(12.5)$ & $2(4.2)$ & $44(25)$ & $5(2.8)$ & $6(6.6)$ & $1(1.1)$ \\
\hline Proteinuria & $16(34.8)$ & $2(4.3)$ & $13(27.7)$ & $1(2.1)$ & $6(12.5)$ & $0(0)$ & $84(47.7)$ & $4(2.3)$ & $15(16.5)$ & $0(0)$ \\
\hline Hypertension & $18(39.1)$ & $5(10.9)$ & $19(40.4)$ & $4(8.5)$ & $2(4.2)$ & $0(0)$ & $62(35.2)$ & $8(4.5)$ & $5(5.5)$ & $0(0)$ \\
\hline Hand foot syndrome & $21(45.7)$ & $6(13)$ & $12(25.5)$ & $2(4.3)$ & $2(4.2)$ & $1(2.1)$ & $49(27.8)$ & $15(8.5)$ & $2(2.2)$ & $0(0)$ \\
\hline Fatigue & $7(15.2)$ & $1(2.1)$ & $8(17)$ & $1(2.1)$ & $5(10.4)$ & $1(2.1)$ & $36(20.5)$ & $5(2.8)$ & $13(14.3)$ & $2(2.2)$ \\
\hline Abdominal pain & $6(13)$ & $1(2.2)$ & $4(8.5)$ & $0(0)$ & $5(10.4)$ & $1(2.1)$ & $28(15.9)$ & $3(1.7)$ & $16(17.6)$ & $4(4.4)$ \\
\hline Diarrhea & $13(28.3)$ & $3(6.5)$ & $8(17)$ & $1(2.1)$ & $2(4.2)$ & $0(0)$ & $20(11.4)$ & $2(1.1)$ & $3(3.3)$ & $1(1.1)$ \\
\hline
\end{tabular}


Table 2. Ongoing trials with Apatinib in gastric cancer.

\begin{tabular}{|c|c|c|c|c|c|c|}
\hline $\begin{array}{l}\text { Clinical trial } \\
\text { identifier }\end{array}$ & Primary Endpoint & Treatment arm & $\begin{array}{l}\text { Patients to be } \\
\text { enrolled }\end{array}$ & Phase & Setting & Status \\
\hline NCT02409199 & $\begin{array}{c}\text { Progression free } \\
\text { survival }\end{array}$ & $\begin{array}{c}\text { Apatinib vs } \\
\text { Docetaxel } \\
\end{array}$ & 66 & II/III & Second line & Recruiting \\
\hline NCT02426034 & Safety and efficacy & Apatinib & 2000 & IV & Second line & $\begin{array}{l}\text { Ongoing, but } \\
\text { not recruiting }\end{array}$ \\
\hline NCT02509806 & $\begin{array}{l}\text { Progression free } \\
\text { survival }\end{array}$ & Apatinib & 48 & & $\begin{array}{l}\text { Maintenance therapy } \\
\text { after first-line }\end{array}$ & $\begin{array}{l}\text { Not yet open for } \\
\text { recruiting }\end{array}$ \\
\hline NCT02510469 & Disease free survival & Apatinib & 48 & & $\begin{array}{c}\text { Maintenance therapy } \\
\text { after adjuvant } \\
\text { chemotherapy }\end{array}$ & $\begin{array}{l}\text { Not yet open for } \\
\text { recruiting }\end{array}$ \\
\hline NCT02525237 & $\begin{array}{l}\text { Progression free } \\
\text { survival; safety }\end{array}$ & $\begin{array}{l}\text { Apatinib } \\
\text { plus S-1 }\end{array}$ & 30 & II & First line & $\begin{array}{l}\text { Not yet open for } \\
\text { recruiting }\end{array}$ \\
\hline NCT02596256 & $\begin{array}{l}\text { Progression free } \\
\text { survival }\end{array}$ & $\begin{array}{c}\text { Apatinib plus } \\
\text { Docetaxel vs } \\
\text { Docetaxel }\end{array}$ & & II & Second line & $\begin{array}{l}\text { Not yet open for } \\
\text { recruiting }\end{array}$ \\
\hline NCT02529878 & Radical resection rate & $\begin{array}{l}\text { S1/Paclitaxel } \\
\text { plus Apatinib }\end{array}$ & 30 & II/III & Conversion therapy* & Recruiting \\
\hline NCT02537171 & $\begin{array}{c}\text { Progression free } \\
\text { survival }\end{array}$ & Apatinib & 40 & III & $\begin{array}{l}\text { Maintenance after first } \\
\text { line treatment }\end{array}$ & Recruiting \\
\hline
\end{tabular}

